\title{
An analytical method with numerical results to be used in the design of optical slab waveguides for optical communication system applications
}

\author{
Aadel M. Alatwi ${ }^{1}$, Ahmed Nabih Zaki Rashed ${ }^{2}$ \\ ${ }^{1}$ Electrical Engineering Department, Faculty of Engineering, Tabuk University, Tabuk, Saudi Arabia \\ ${ }^{2}$ Electronics and Electrical Communications Engineering Department, Faculty of Electronic Engineering, \\ Menoufia University, Menofia Governorate, Egypt
}

\begin{tabular}{l}
\hline \hline Article Info \\
\hline Article history: \\
Received Mar 26, 2020 \\
Revised Jun 27, 2020 \\
Accepted Jul 13, 2020 \\
\hline
\end{tabular}

\section{Keywords:}

Analytical methods Numerical schemes Slab waveguide Waveguide simulation

\begin{abstract}
This study develops an analytical method with numerical results for the design of optical slab waveguides for optical communication system applications. An optical slab waveguide structure made of silicon on silicon dioxide material is designed and analyzed. The effective index of the mode is studied against variations in the waveguide dimensions. Transmission and reflection coefficients are studied and compared to the wavelength and dimensions of the waveguide. Variations are sketched with the $\mathrm{x}$-axis, in addition to the electric field intensity distribution and effective refractive index. Waveguide bending loss is also studied with waveguide thickness and length variations within three waveguide transmission windows of 850 $\mathrm{nm}, 1300 \mathrm{~nm}$, and $1550 \mathrm{~nm}$.
\end{abstract}

This is an open access article under the CC BY-SA license.

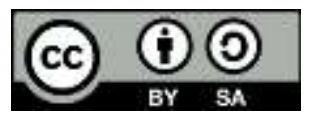

Corresponding Author:

Ahmed Nabih Zaki Rashed

Faculty of Electronic Engineering

Menoufia University, Menouf, Egypt

Email:_ahmed_733@yahoo.com

\section{INTRODUCTION}

A uniform dielectric waveguide is the most basic structure in fiber and integrated optics. Therefore, the calculation of the modal characteristics of uniform dielectric waveguides is a fundamental research topic $[1,2]$. Since so many important physical similarities exist between dielectric and metal waveguides, it seems that the well-established methods for analyzing metallic waveguides can be easily and directly applied to dielectric waveguides [3-5]. However, this is rarely the case. It may happen that a method that is highly successful for metallic waveguides may become unsuitable, if not useless, for the dielectric counterparts. This is particularly true for analytical methods. For example, the method of separation of variables applies remarkably well to a rectangular metallic waveguide but fails for a rectangular dielectric rod because of the presence of interfaces between different dielectrics [2,6].

The analytical results are far more complicated than those for the circular metallic tube, even for the familiar circular step-index fiber [1, 7, 8]. Dielectric waveguides are, indeed, much more difficult to analyze analytically. Moreover, optical waveguide technology has advanced to a stage of high sophistication; therefore, scientists and engineers must study more complex and general waveguide structures for which analytical solutions simply do not exist. It is necessary to construct good numerical methods that can handle very general optical waveguides. In the meantime, the rapid growth of computer technology has also popularized the application of numerical methods [1, 4, 7-9]. 
To a large extent, the numerical methods used to analyze dielectric waveguides are basically those that have been widely applied to hollow metallic waveguides, for example, the finite element method and the finite difference method [1, 4, 7, 10,11]. However, the existence of hybrid modes in a dielectric waveguide has complicated the formulation of these conventional methods significantly, and accurate modeling of the open region of the waveguide has further reduced their computational efficiency. Research efforts have been directed to either invent completely new numerical methods specifically for dielectric waveguides or modify existing methods to circumvent some or all of their disadvantages. The effective index method is one of these new methods, and the finite element method is the most extensively adapted method $[2,7,9,12]$.

In $[1,2,7,13]$, using a perturbation method, has derived an accurate explicit far from cutoff formula for TE modes of symmetric three-layer isotropic slab waveguides. Their formula is generalized to account for both TE and TM modes of isotropic and anisotropic waveguides. However, it is found that the accuracy of the formula for TM modes in the low-frequency range decreases significantly with increasing slab-cladding index ratio. A close to a cutoff formula is then derived to complement the far from the cutoff formula.

\section{MODEL DESCRIPTION AND RESEARCH METHOD}

An optical slab waveguide is constructed using a silicon material doped with germanium material with a refractive index of $\mathrm{n}_{1}$. The available doping ratio of germanium is added to the silicon material up to $30 \%$ in order to upgrade the waveguide transmission performance. The optical slab waveguide configuration is rectangular and $b$ is the length, $\lambda$ is the waveguide wavelength, and $t$ is the optical waveguide thickness. The surrounding regions along the optical slab waveguide are made of silicon dioxide material, which acts as an insulator that has a refractive index of $\mathrm{n}_{2}$. The other regions consist of free space (air) with a refractive index of $\mathrm{n}_{0}$. Optical slab waveguide as shown in Figure 1.



Figure 1. Optical slab waveguide structure made of silicon on silicon dioxide material

The relationship between the index of refraction for the core layer and the index of refraction for the cladding layer can be classified using Snell's law, if total internal reflection occurs [1, 2, 7-12]:

$$
n_{1} \sin \theta_{1}=n_{2} \sin \theta_{2}
$$

If total internal reflection occurs and $\theta_{2}=90$ degrees, then the critical angle will be:

$$
\theta_{c}=\sin ^{-1}\left(\frac{n_{2}}{n_{1}}\right)
$$

where the multiplication of the electric field and magnetic field can be expressed in terms of permittivity and permeability as [1, 2, 13-17]:

$$
S=E \times H=\sqrt{\frac{\varepsilon_{m}}{\mu_{m}}} E^{2}
$$

where the refection coefficient $(r)$ for the transverse electric and transverse magnetic are given by [1, 7-9]: 


$$
\begin{aligned}
& r_{T E}=\frac{n_{1} \cos \left(\theta_{1}\right)-\sqrt{n_{2}^{2}-n_{1}^{2} \sin ^{2}\left(\theta_{1}\right)}}{n_{1} \cos \left(\theta_{1}\right)+\sqrt{n_{2}^{2}-n_{1}^{2} \sin ^{2}\left(\theta_{1}\right)}} \\
& r_{T M}=\frac{n_{2}^{2} \cos \left(\theta_{1}\right)-n_{1} \sqrt{n_{2}^{2}-n_{1}^{2} \sin ^{2}\left(\theta_{1}\right)}}{n_{2}^{2} \cos \left(\theta_{1}\right)+n_{1} \sqrt{n_{2}^{2}-n_{1}^{2} \sin ^{2}\left(\theta_{1}\right)}}
\end{aligned}
$$

where the transmission coefficient $(\mathrm{T})$ can be given as a basic main function of reflected coefficient ( $\mathrm{r}$ ) as:

$$
T=1-r
$$

where the transverse magnetic mode via a slab optical waveguide analysis [1, 2, 16, 18-22]:

$$
\frac{\sqrt{\sin ^{2}\left(\theta_{1}\right)-\left(\frac{n_{1}}{n_{2}}\right)^{2}}}{\cos \left(\theta_{1}\right)}=\tan \left(\frac{k_{0} n_{1} \cos \left(\theta_{1}\right)-m \pi}{2}\right)
$$

In addition, the wave number in the optical slab waveguide is given by:

$$
k_{o}=\frac{2 \pi}{\lambda_{o}}
$$

where $\lambda_{0}$ is the waveguide wavelength. Where the waveguide length is $b$ and the waveguide width is a, where the ratio between waveguide width and waveguide length is expressed by [1, 2, 7-10]:

$$
\begin{aligned}
& \frac{a}{b} \leq\left(\frac{q+4 \pi b}{4 \pi b}\right) \frac{1+0.3 \sqrt{\left(\frac{q+4 \pi b}{q+4 \pi r b}\right)^{2}-1}}{\sqrt{\left(\frac{q+4 \pi b}{q+4 \pi r b}\right)^{2}-1}} \\
& q=\frac{\gamma_{0}}{\sqrt{n_{1}^{2}-n_{0}^{2}}}+\frac{\gamma_{2}}{\sqrt{n_{1}^{2}-n_{2}^{2}}}
\end{aligned}
$$

where the bending radius of the optical slab waveguide can be expressed for the bend loss coefficient factor as $[1,2,18,23-25]$ :

$$
\alpha_{\text {bend }}=C_{1} e^{-C_{2} R}
$$

where the basic constants $\mathrm{C}_{1}, \mathrm{C}_{2}$, and $\mathrm{R}$ is the bending radius of the slab waveguide are calculated by $[1,2,4,19]$ :

$$
\begin{aligned}
& C_{1}=\frac{\lambda_{0} \cos ^{2}\left(k_{x g} \frac{w}{2}\right) e^{k_{x s} w}}{w^{2} k_{x s} n_{e f f c}\left(\frac{w}{2}+\frac{1}{2 k_{x g}} \sin \left(w k_{x g}\right)+\frac{1}{k_{x s}} \cos ^{2}\left(k_{x g} \frac{w}{2}\right)\right)} \\
& C_{2}=2 k_{x s}\left(\frac{\lambda_{0} \beta}{2 \pi n_{e f f c}}-1\right)
\end{aligned}
$$


Where $\beta$ is the propagation constant, $\mathrm{n}_{\mathrm{effc}}$ is the effective cladding refractive index, and $\mathrm{k}_{\mathrm{xs}}$ is the decay or delay coefficient, and where the coupling coefficient for the different modes in the optical slab waveguide can be estimated by $[2,7,9,20,21]$ :

$$
\kappa=\frac{2 k_{x c}^{2} k_{x s} e^{-k_{x s} s}}{\beta w\left(k_{x s}^{2}+k_{x c}^{2}\right)}
$$

where the propagation constant $\beta$ can be estimated by $[1,2,7-12,26-28]$ :

$$
\beta=\beta_{r}-i \frac{\alpha_{\text {loss }}}{2}
$$

where $\alpha_{\text {loss }}$ is the loss coefficient and $\beta_{\mathrm{r}}$ is the reflection propagation constant, and where the core refractive index is given by the following equation $[1,2,4,7-10]$ :

$$
n_{1}=\sqrt{\frac{A_{1}-B_{1} \lambda}{C_{1}^{2}-\lambda^{2}}}
$$

where the constants are $A_{1}=0.0675 x, B_{1}=2+0.012 x$, and $C_{1}=1.32 \mathrm{x}+0.54$. Moreover, $\mathrm{x}$ is the germanium dopant ratio that is added to the silicon fabrication material.

\section{PERFORMANCE ANALYSIS WITH DISCUSSIONS}

The effective index method was originally constructed to handle rectangular dielectric structures that are encountered in millimeter-wave transmission and integrated optics. The method aims to replace the approximate rectangular structure by two planar waveguides. This approach facilitates computation and provides an intuitive understanding of the modal properties of the waveguide. In fact, it is the simplest and most efficient method available for analyzing general rectangular dielectric waveguides. Table 1 lists the operating parameters for the slab rectangular waveguide.

Table 1. Parameters used in the rectangular slab waveguide

\begin{tabular}{cc}
\hline Parameters & Value/unit \\
\hline Waveguide thickness, $\mathrm{t}$ & $10 \mathrm{~nm}-30 \mathrm{~nm}$ \\
Waveguide length, $\mathrm{b}$ & $0.2 \mu \mathrm{m}-1.6 \mu \mathrm{m}$ \\
Waveguide width, a & $0.1 \mu \mathrm{m}-0.3 \mu \mathrm{m}$ \\
Waveguide wavelength & $850 \mathrm{~nm}-1650 \mathrm{~nm}$ \\
Ge dopant ratio, $\mathrm{x}$ & $2 \%-30 \%$ \\
\hline
\end{tabular}

Figure 2 clarifies the mode effective index variations with the waveguide length and thickness at an operating transmission wavelength of $850 \mathrm{~nm}$. As the waveguide thickness increases, the mode effective index increases. But as the waveguide length increases, mode effective index decreases. With a waveguide thickness of $30 \mathrm{~nm}$ and a waveguide length of $0.2 \mu \mathrm{m}$, the mode effective index is 1.521 . With a waveguide thickness of $30 \mathrm{~nm}$ and a waveguide length of $0.8 \mu \mathrm{m}$, the mode effective index is 1.155 . However, with a waveguide thickness of $30 \mathrm{~nm}$ and waveguide length of $1.6 \mu \mathrm{m}$, the mode effective index is 0.853 .

Figure 3 indicates the mode effective index variations with the waveguide length and thickness with an operating transmission wavelength of $1300 \mathrm{~nm}$. As the waveguide thickness increases, mode effective index increases. However, as the waveguide length increases, the mode effective index decreases. With a waveguide thickness of $30 \mathrm{~nm}$ and a waveguide length of $0.2 \mu \mathrm{m}$, the mode effective index is 1.62 . With a waveguide thickness of $30 \mathrm{~nm}$ and a waveguide length of $0.8 \mu \mathrm{m}$, the mode effective index is 1.332 . However, with a waveguide thickness of $30 \mathrm{~nm}$ and waveguide length of $1.6 \mu \mathrm{m}$, the mode effective index is 0.889 . Figure 4 shows the mode effective index variations with waveguide length and thickness at an operating transmission wavelength of $1550 \mathrm{~nm}$. As the waveguide thickness increases, the mode effective index increases. However, as the waveguide length increases, the mode effective index decreases. With a waveguide thickness of $30 \mathrm{~nm}$ and a waveguide length of $0.2 \mu \mathrm{m}$, the mode effective index is 1.702 . With a waveguide thickness of $30 \mathrm{~nm}$ and a waveguide length of $0.8 \mu \mathrm{m}$, the mode effective index is 1.456 . However, with a waveguide thickness of $30 \mathrm{~nm}$ and a waveguide length of $1.6 \mu \mathrm{m}$, the mode effective index is 0.976 .

An analytical method with numerical results to be used in the design of optical slab... (Aadel M. Alatwi) 


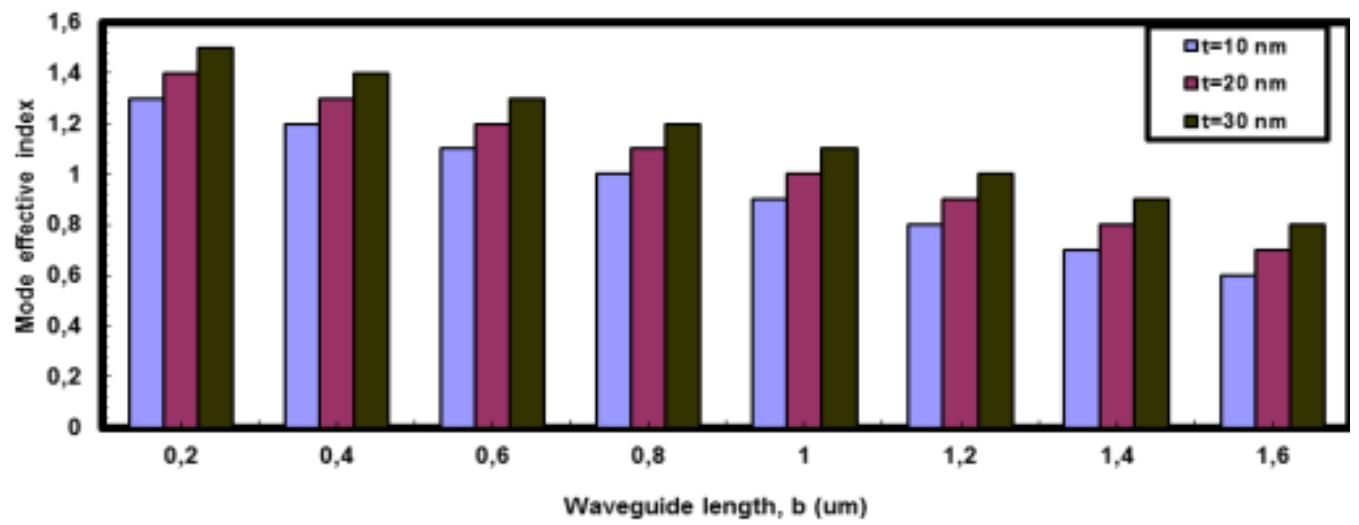

Figure 2. Mode effective index variations versus waveguide length and thickness at an operating wavelength of $850 \mathrm{~nm}$

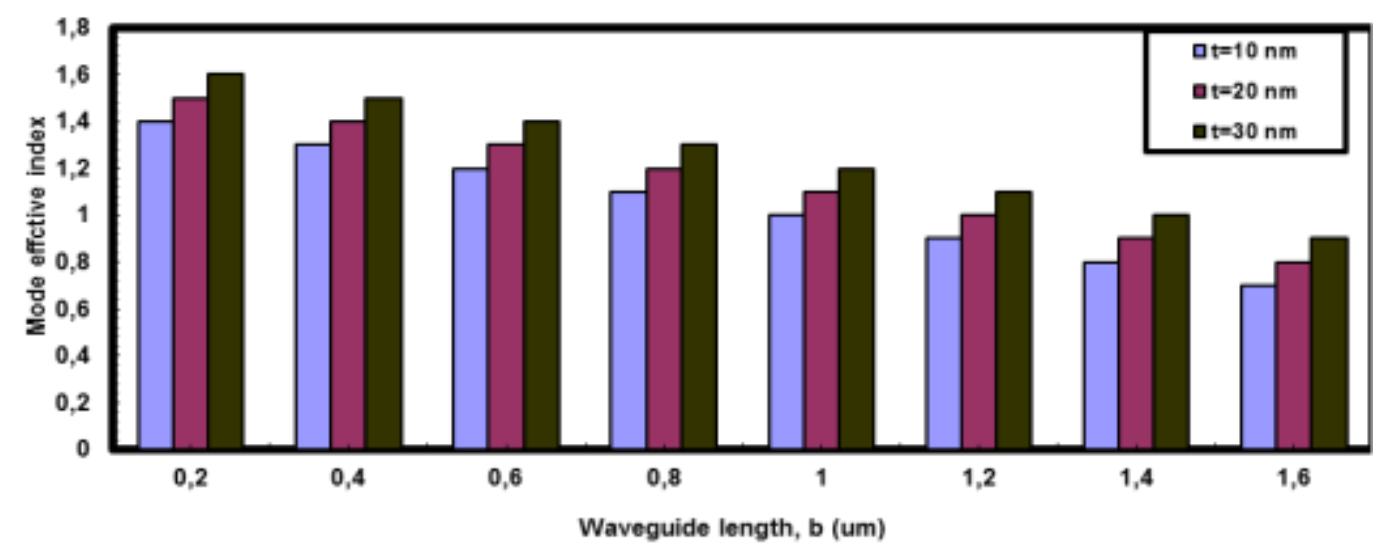

Figure 3. Mode effective index variations versus waveguide length and thickness at an operating wavelength of $1300 \mathrm{~nm}$

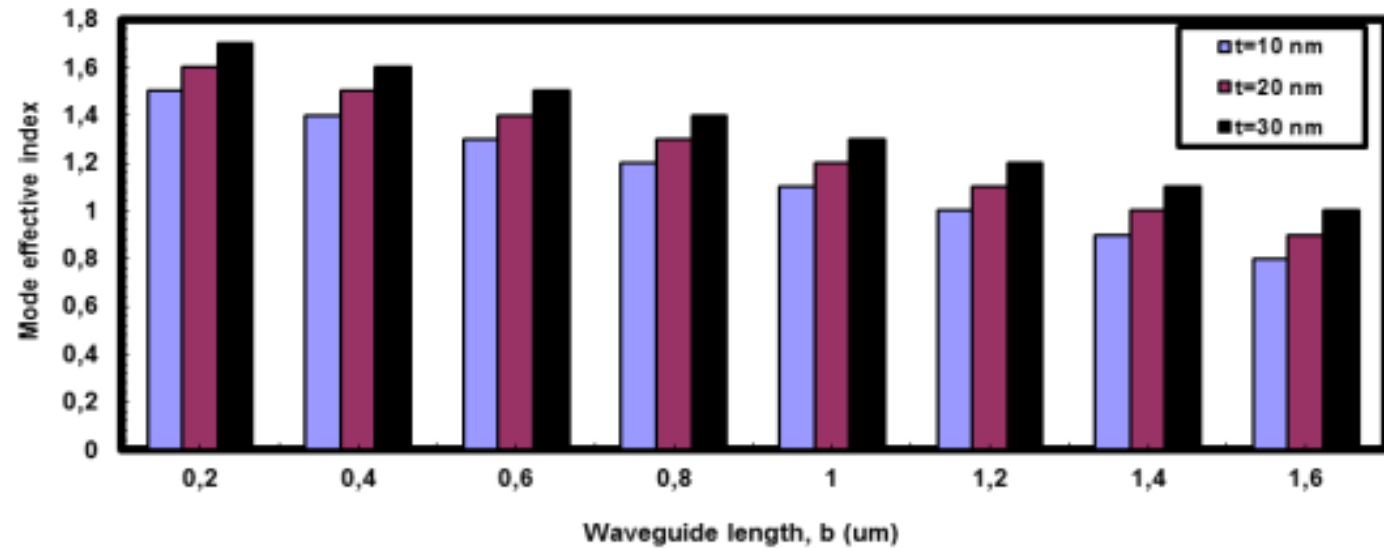

Figure 4. Mode effective index variations versus waveguide length and thickness at an operating wavelength of $1550 \mathrm{~nm}$

Figure 5 shows the transmission coefficient variations with waveguide wavelength and thickness variations. As the waveguide wavelength and width increase, the waveguide light transmission coefficient also increases. With a waveguide width value of $0.3 \mu \mathrm{m}$ and a waveguide wavelength value of $850 \mathrm{~nm}$, the 
waveguide light transmission coefficient is 0.7795 . With a waveguide width value of $0.3 \mu \mathrm{m}$ and a waveguide wavelength value of $1300 \mathrm{~nm}$, the waveguide light transmission coefficient is 0.8865 . Moreover, with a waveguide width value of $0.3 \mu \mathrm{m}$ and a waveguide wavelength value of $1550 \mathrm{~nm}$, the waveguide light transmission coefficient is 0.995 .

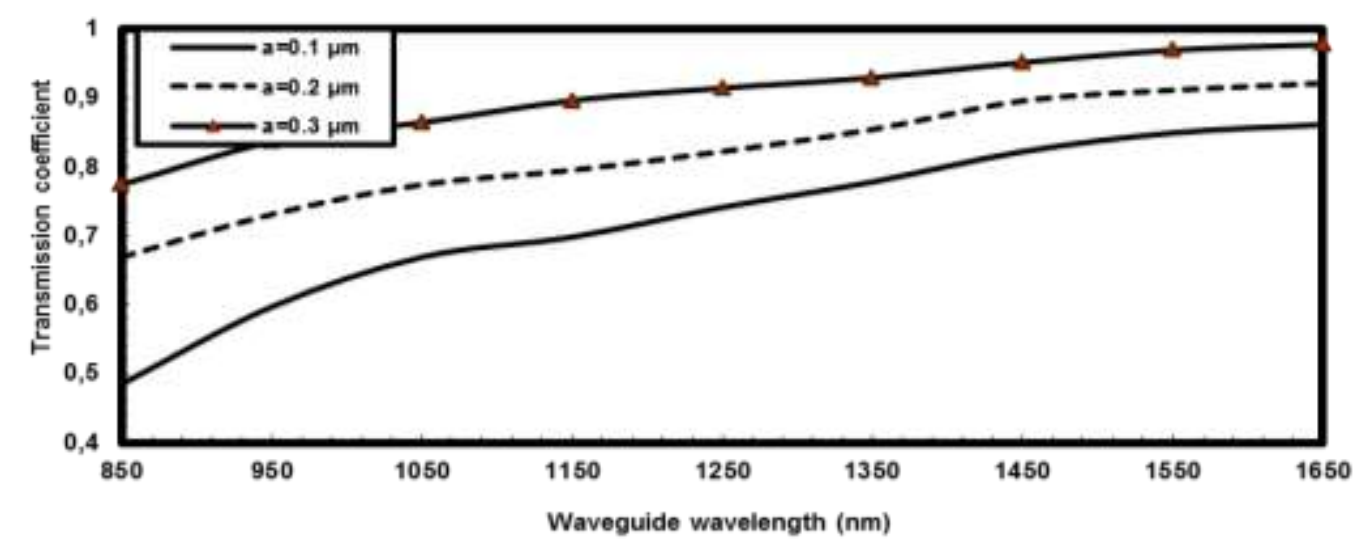

Figure 5. Transmission coefficient variations with waveguide wavelength and thickness variations

Figure 6 shows the reflection coefficient variations with waveguide wavelength and thickness variations. As the waveguide wavelength and width increases, the waveguide light reflection coefficient decreases. With a waveguide width value of $0.3 \mu \mathrm{m}$ and a waveguide wavelength value of $850 \mathrm{~nm}$, the waveguide light transmission coefficient is 0.3231 . Moreover, with a waveguide width value of $0.3 \mu \mathrm{m}$ and a waveguide wavelength value of $1300 \mathrm{~nm}$, the waveguide light transmission coefficient is 0.1233 . However, with a waveguide width value of $0.3 \mu \mathrm{m}$ and a waveguide wavelength value of $1550 \mathrm{~nm}$, the waveguide light reflection coefficient is 0.005 .

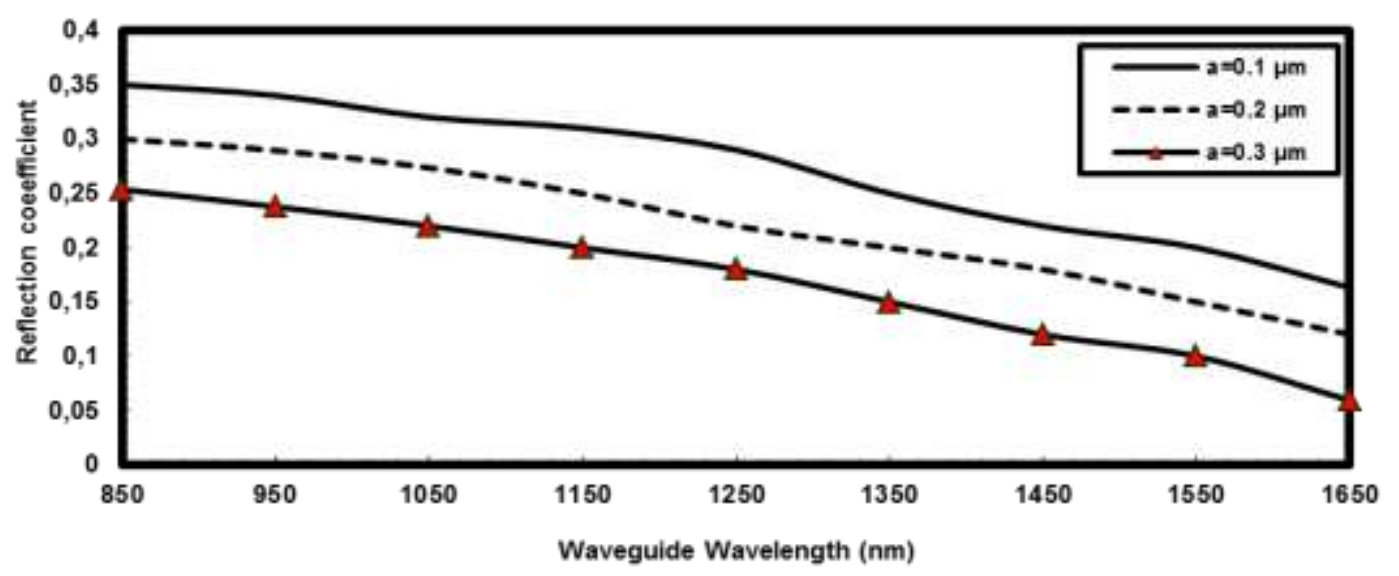

Figure 6. Reflection coefficient variations with waveguide wavelength and thickness variations

Figure 7 shows the electric field intensity and effective refractive index with x-axis distribution. This figure shows that the electric field intensity has reached a value of 0.8 and an effective refractive index value of 1.6. Figure 8 shows the waveguide bending loss variations with waveguide length and thickness at an operating wavelength of $1550 \mathrm{~nm}$. With a waveguide thickness value of $10 \mathrm{~nm}$ and a waveguide length value of $0.2 \mu \mathrm{m}$, the waveguide bending loss is $0.022 \times 10^{-4} \mathrm{~dB} / \mu \mathrm{m}$. With a waveguide thickness value of 10 $\mathrm{nm}$ and a waveguide length value of $0.8 \mu \mathrm{m}$, the waveguide bending loss is $0.085 \times 10^{-4} \mathrm{~dB} / \mu \mathrm{m}$. However, with a waveguide thickness value of $10 \mathrm{~nm}$ and a waveguide length value of $1.6 \mu \mathrm{m}$, the waveguide bending loss value is $0.136 \times 10^{-4} \mathrm{~dB} / \mu \mathrm{m}$. 
Figure 9 shows the waveguide bending loss variations with waveguide length and thickness at an operating wavelength of $1300 \mathrm{~nm}$. With a waveguide thickness value of $10 \mathrm{~nm}$ and a waveguide length value of $0.2 \mu \mathrm{m}$, the waveguide bending loss is $0.041 \times 10^{-4} \mathrm{~dB} / \mu \mathrm{m}$. Moreover, with a waveguide thickness value of $10 \mathrm{~nm}$ and a waveguide length value of $0.8 \mu \mathrm{m}$, the waveguide bending loss is $0.065 \times 10^{-4} \mathrm{~dB} / \mu \mathrm{m}$. However, with a waveguide thickness value of $10 \mathrm{~nm}$ and a waveguide length value of $1.6 \mu \mathrm{m}$, the waveguide bending loss value is $0.135 \times 10^{-4} \mathrm{~dB} / \mu \mathrm{m}$. Figure 10 indicates the waveguide bending loss variations with a waveguide length and thickness at an operating wavelength of $850 \mathrm{~nm}$. With a waveguide thickness value of $10 \mathrm{~nm}$ and a waveguide length value of $0.2 \mu \mathrm{m}$, the waveguide bending loss value is $0.062 \times 10^{-4} \mathrm{~dB} / \mu \mathrm{m}$. Mover, with a waveguide thickness value of $10 \mathrm{~nm}$ and a waveguide length value of $0.8 \mu \mathrm{m}$, the waveguide bending loss value is $0.094 \times 10^{-4} \mathrm{~dB} / \mu \mathrm{m}$. Therefore, with a waveguide thickness value of $10 \mathrm{~nm}$ and a waveguide length value of $1.6 \mu \mathrm{m}$, the waveguide bending loss is $0.142 \times 10^{-4} \mathrm{~dB} / \mu \mathrm{m}$.

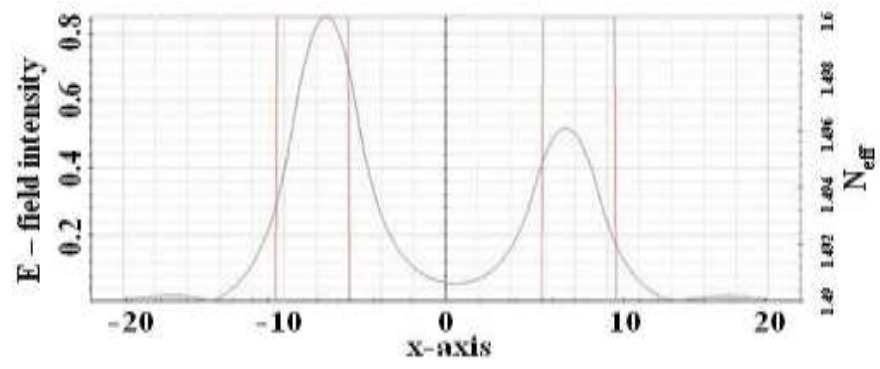

Figure 7. Electric field intensity and effective refractive index with x-axis distribution

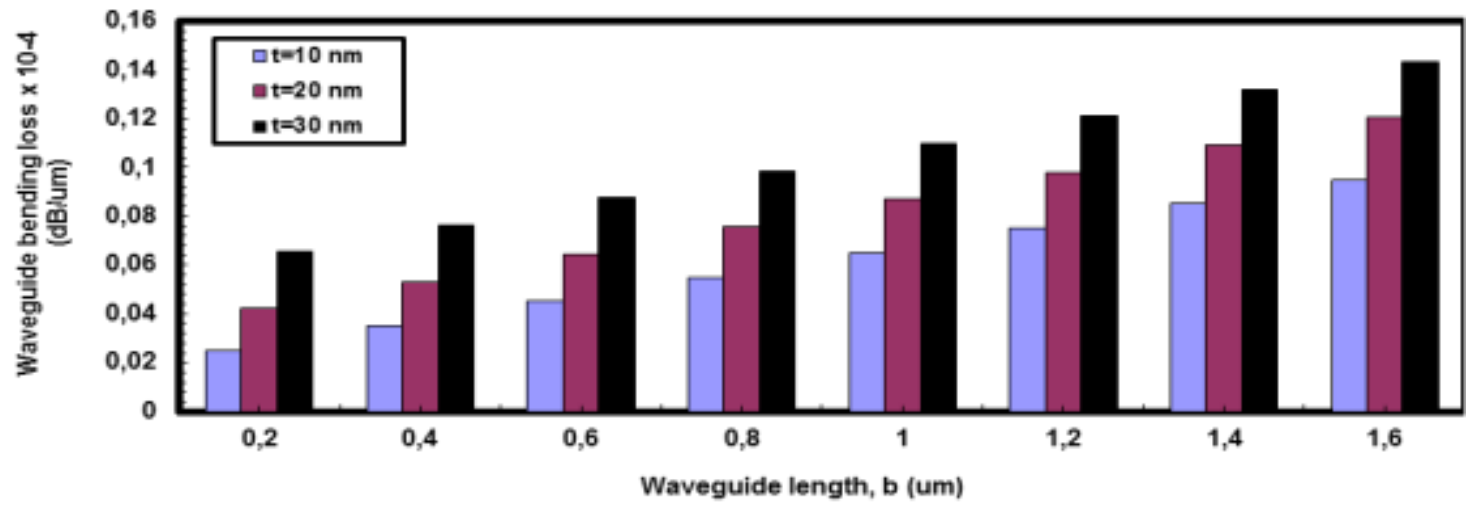

Figure 8 . Waveguide bending loss variations with waveguide length and thickness at an operating wavelength of $1550 \mathrm{~nm}$

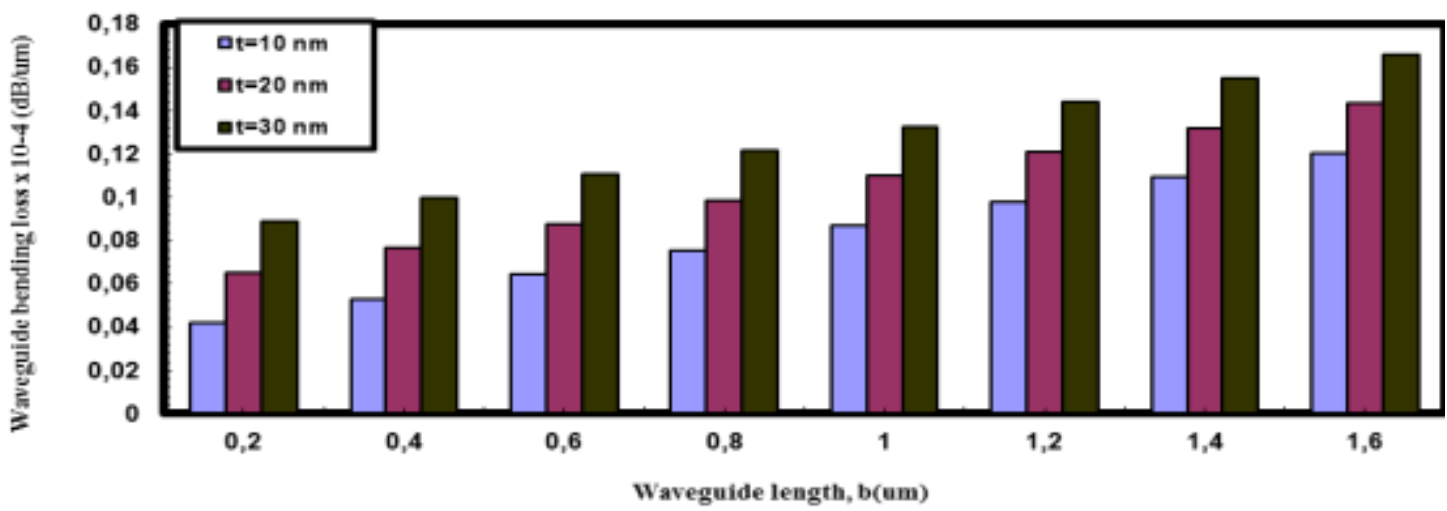

Figure 9. Waveguide bending loss variations with waveguide length and thickness at an operating wavelength of $1300 \mathrm{~nm}$ 


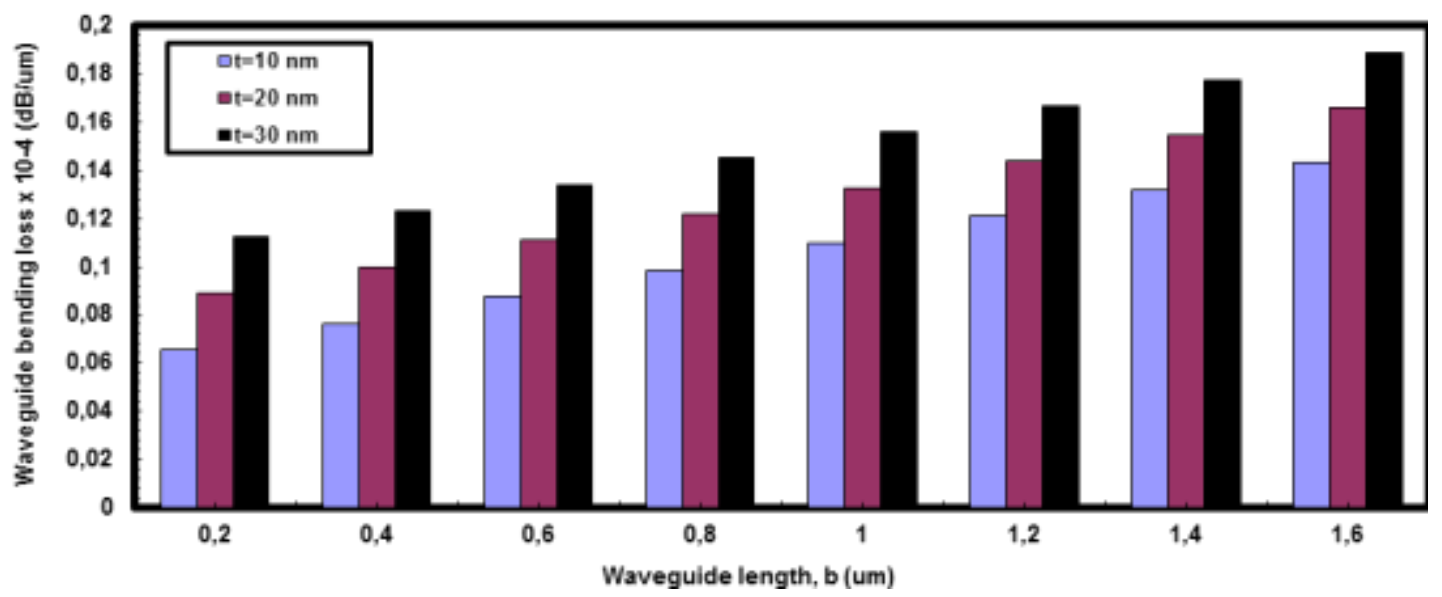

Figure 10. Waveguide bending loss variations with waveguide length and thickness at an operating wavelength of $850 \mathrm{~nm}$

Figure 11 shows the transmission and reflection coefficients versus the germanium dopant ratio at an operating wavelength of $1550 \mathrm{~nm}$. As the germanium dopant ratio increases, the transmission coefficient increases and the reflection coefficient decreases. Moreover, with a dopant ratio of $2 \%$, the average transmission coefficient reaches 0.55 while the average reflection coefficient reaches 0.45 . With a dopant ratio of $16 \%$, the average transmission coefficient reaches 0.85 and the average reflection coefficient reaches 0.15 . Finally, with a dopant ratio of $30 \%$, the average transmission coefficient reaches 0.95 and the average reflection coefficient reaches 0.05 .

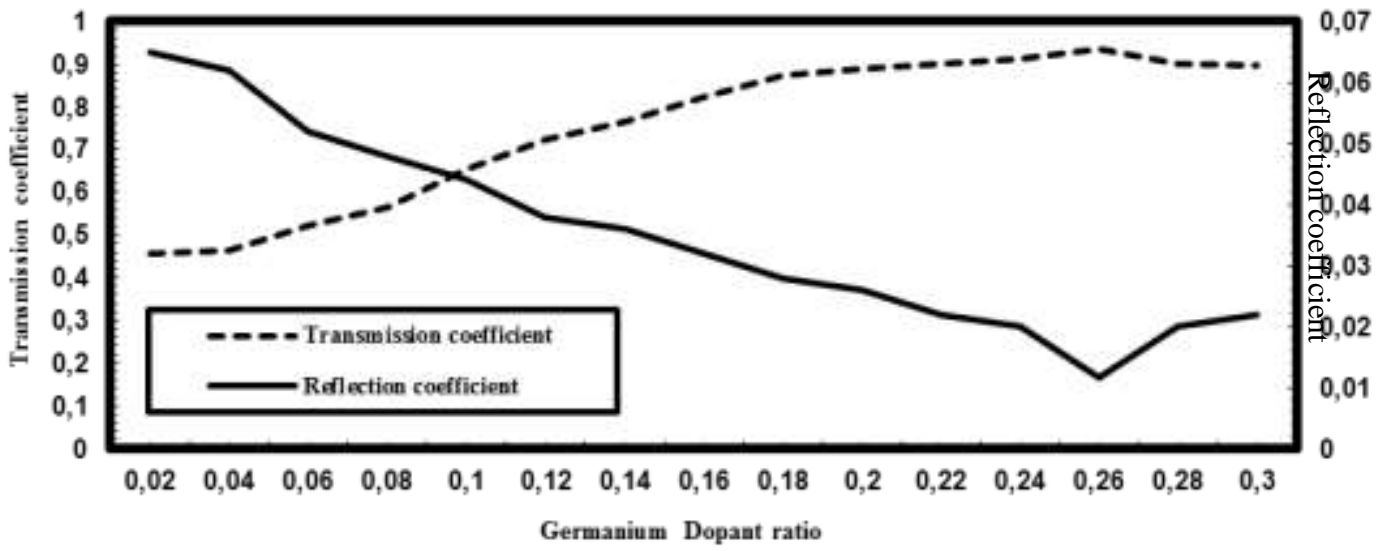

Figure 11. Transmission and reflection coefficients versus the germanium dopant ratio at an operating wavelength of $1550 \mathrm{~nm}$

\section{CONCLUSION}

The design of the optical slab waveguide is designed and analyzed within three optical transmission windows of $850 \mathrm{~nm}, 1300 \mathrm{~nm}$, and $1550 \mathrm{~nm}$. The waveguide bending loss can be optimized at a waveguide length of $0.2 \mu \mathrm{m}$ and a waveguide thickness of $10 \mathrm{~nm}$. Therefore, the optimum waveguide bending loss value is $0.022 \times 10^{-4}$ $\mathrm{dB} / \mu \mathrm{m}$. The electric field intensity distribution is 0.8 and the effective refractive index value is 1.6 . In addition to the waveguide light transmission, the coefficient is 0.995 at a waveguide width of $0.3 \mu \mathrm{m}$ and a waveguide wavelength of $1550 \mathrm{~nm}$. The optimized waveguide light reflection coefficient is 0.005 at a waveguide width value of $0.3 \mu \mathrm{m}$ and a waveguide wavelength value of $1550 \mathrm{~nm}$. In the same way, the optimized mode effective index is reached at 0.976 with a waveguide thickness of $30 \mathrm{~nm}$ and a waveguide length of $1.6 \mu \mathrm{m}$. 


\section{REFERENCES}

[1] S. E. Miller and I. P. Kaminow, “Optical Fiber Telecommunications II," Academic Press, 1988.

[2] D. Marcuse, "Principle of optical fiber measurements," Academic Press, 1981.

[3] I. S. Amiri and A. N. Z. Rashed, "Numerical investigation of V shaped three elements resonator for optical closed loop system," Indonesian Journal of Electrical Engineering and Computer Science, vol. 16, no. 3, pp. 1392-1397, 2019.

[4] A. J. Danner, et al., "Single fundamental mode photonic crystal vertical cavity laser with improved output power," Electronics Letters, vol. 41, pp. 325-326, 2005.

[5] A. N. Z. Rashed, et al., "Different Pumping Categories of Erbium Doped Fiber Amplifiers Performance Signature with Both Wide Multiplexing and Modulation Techniques," International Journal of Science, Engineering and Technology Research (IJSETR), vol. 5, no. 3, pp. 622-642, 2016.

[6] A. N. Z. Rashed et al., "Performance Evaluation of SAC-OCDMA System in Free Space Optics and Optical Fiber System Based on Different Types of Codes," Wireless Personal Communications Journal, vol. 96, no. 2, pp. 2843-2861, 2017.

[7] Y. Kaneko, et al., "Transverse-mode characteristics of InGaA/GaAs vertical-cavity surface-emitting lasers considering gain offset," Japanese Journal of Applied Physics, vol. 32, pp. 1612-1614, 1993.

[8] E. F. Schubert, et al., "Temperature and Modulation Characteristics of Resonant-Cavity Light-Emitting Dioded," IEEE Journal of Lightwave Technology, vol 14, pp. 1721-1728, 1996.

[9] D. G. Deppe, et al., "Optically-coupled Mirror-Quantum Well InGaAs-GaAs Light Emitting Diode," Electronics Letter, vol. 26, pp. 1665-1666, 1990.

[10] N. Debbar, et al., "Coupled GaAs/AlGaAs quantum-well electro absorption modulators for low-electric-field optical modulation,” J. Appl. Phys., vol. 65, pp. 383-385, 1989.

[11] V. Kumar and K. Singh, "Model for calculating the refractive index of different materials," Indian journal of pure and applied physics, vol. 48, no. 8, pp. 571-574, 2010.

[12] J. Nagel, et al., "High Power, Narrow Linewidth Continuous Wave Raman Amplifier at $1.27 \mu \mathrm{m}$," IEEE Photonics Technology Letters, vol. 23, no. 9, pp. 453-466, 2011.

[13] A. N. Z. Rashed, et al., "Optical Add Drop Multiplexers with UW-DWDM Technique in Metro Optical Access Communication Networks," Nonlinear Optics and Quantum Optics, vol. 44, no. 1, pp. 25-39, 2012.

[14] A. N. Z. Rashed, et al., "High Transmission Performance of Radio over Fiber Systems over Traditional Optical Fiber Communication Systems Using Different Coding Formats for Long Haul Applications," Nonlinear Optics and Quantum Optics, vol. 44, no. 1, pp. 41-63, 2012.

[15] H. B. Sharma, et al., "Evaluation of Optical Amplifiers," International Journal of Engineering Research and Applications (IJERA), vol. 2, no. 1, pp. 663-667, 2012.

[16] Q. Hen, et al., "Novel Shooting Algorithm for Highly Efficient Analysis of Fiber Raman Amplifiers," IEEE J. Lightwave Technol., vol. 24, no. 4, pp. 1946-1952, 2006.

[17] I. S. Amiri, et al., "Temperature effects on characteristics and performance of near-infrared wide bandwidth for different avalanche photodiodes structures," Results in Physics, vol. 14, 2019.

[18] I. S. Amiri and A. N. Z. Rashed, "Simulative study of simple ring resonator-based brewster plate for power system operation stability," Indonesian Journal of Electrical Engineering and Computer Science, vol. 16, no. 2, pp. 1070-1076, 2019.

[19] A. N. Z. Rashed and M. S. F. Tabbour, "Suitable Optical Fiber Communication Channel for Optical Nonlinearity Signal Processing in High Optical Data Rate Systems," Wireless Personal Communications Journal, vol. 97, no. 1, pp. 397-416, 2017.

[20] A. N. Z. Rashed, et al., "Optimum Flat Gain With Optical Amplification Technique Based on Both Gain Flattening Filters and Fiber Bragg Grating Methods," Journal of Nanoelectronics and Optoelectronics, vol. 13, no. 5, pp. 665-676, 2018.

[21] A. N. Z. Rashed, et al., "The switching of optoelectronics to full optical computing operations based on nonlinear metamaterials," Results in Physics, vol. 13, 2019.

[22] A. N. Z. Rashed, et al., "Design of D-shaped elliptical core photonic crystal fiber for blood plasma cell sensing application," Results in Physics, vol. 12, pp. 2021-2025, 2019.

[23] A. N. Z. Rashed, et al., "Performance enhancement of IM/DD optical wireless systems," Photonic Network Communications, vol. 36, no. 1, pp. 114-127, 2018.

[24] I. S. Amiri, et al., "Spatial Continuous Wave Laser and Spatiotemporal VCSEL for High-Speed Long Haul Optical Wireless Communication Channels," Journal of Optical Communications, 2019.

[25] I. S. Amiri, et al., "Influence of Loading, Regeneration and Recalling Elements Processes on the System Behavior of All Optical Data Bus Line System Random Access Memory," Journal of Optical Communications, 2019.

[26] I. S. Amiri, et al., "The Engagement of Hybrid Ultra High Space Division Multiplexing with Maximum Time Division Multiplexing Techniques for High-Speed Single-Mode Fiber Cable Systems," Journal of Optical Communications, 2019.

[27] I. S. Amiri, et al., "Performance Enhancement of Fiber Optic and Optical Wireless Communication Channels by Using Forward Error Correction Codes," Journal of Optical Communications, 2019.

[28] I. S. Amiri, et al., "Comparative Simulation Study of Multi Stage Hybrid All Optical Fiber Amplifiers in Optical Communications," Journal of Optical Communications, 2020. 